\title{
Analysis of Communication Radius Configuration Scheme with Double-chain Linear Topology Network
}

\author{
Sai Wu, Xikui Gao, Yan Bai, Shuai Gao \\ (School of Control and Computer Engineering, North China Electric Power University, 102206, China) \\ wusai2008@163.com
}

\begin{abstract}
For railways, bridges, highways and others constructed by fixed-chain type linear wireless sensor networks, many studies have focused on routing protocol research with stetted communication radius. However, different communication radius directly impacts the transmission; thereby affects the performance of the network lifetime and protocols. To solve this problem, this paper models the impact of changes in the communication radius on network performance. First, the lifetime and latency model of double-chain linear topology network are proposed, the effect process of changes in the communication radius on the network life, network delay is detailed analyzed, and then optimal communication radius algorithm is put forward based on delay tolerance. Further analysis is concerned on the effect of different network widths, length and scale to the optimal communication radius. Configuration scheme of communication radius is proposed under different network structures in order to maximize network lifetime as the optimization target. Finally the effectiveness of the proposed model and scheme are verified by theoretical and simulation analysis. It is meaningful for configure reasonable communication radius at different real project.
\end{abstract}

Keywords-Siphonic: Double-chain Type; Linear Topology Network; Communication Radius; Network Lifetim; Network Time Delay; Network Structure

\section{INTRODUCTION}

Because of small size, low cost, and easy deployment, wireless sensor network (WSN) has been widely used to monitor data for railways, bridges, highways, and others which is hardly to use wire to communication ${ }^{[1]}$. These special areas constitute double-chain linear wireless sensor networks. Under normal circumstances, using batterypowered wireless sensors results in end nodes of linear network excessive energy consumption, and the initial node problem of excessive delay. Many studies have focused on the study of routing protocol with communication radius value settled. However Communication radius determined by the transmission power ${ }^{[2]}$ directly impacts the transmission of networks, thereby affecting the life and performance of the network protocol in good or bad state. Selecting the appropriate communication radius can reduce the network delay, improve network lifetime, and improving the network performance. Therefore it is significant to analyze the communication radius.

There are many aspects of current research for the linear network topology. Zhu Y H[3] proposed a method using linear programming and dynamic routing to extend the network lifetime. Hossain $\mathrm{A}^{[4]}$ sought the optimal spacing and optimum density under the circumstance of seeking the optimal spacing with fixed node position, and finding the optimal density of nodes with fixed spacing. Wang $Z^{[5]}$ put forward an energy balanced deployment strategy for strip-based wireless sensor networks. It has been used to analyze the impact of network structure on optimal node spacing. To simplify the transmission model, above-mentioned methods assumed the communication radius meeting the requirements no matter under singlehop or multi-hop, they did notcarefully consider the effects of different communication radius on the algorithms.

For communication radius, there are many aspects of research. Mao $\mathrm{J}^{[6]}$ presented the transmission radius changes affecting the single-hop distance and capacity, and then presented to the relationship with network capacity. Shelby $Z^{[7]}$ proposed a relationship between the transmission radius and sensor network performance. By analyzing the transmission radius affects to hops from source node to destination node, the author presents the result that six adjacent nodes controlled by transmission radius were best. Dong $\mathrm{P}^{[8]}$ analyzed the impact of network communication radius on the energy consumption to obtain two energy consumption comparison chart in the case of the node density infinite or less than infinity, and the result show communication radius was affected by node density and node spacing. However, the model was obtained in single-link analysis model, so it wasn't available under the double-link model.

The above-mentioned documents didn't consider the communication radius impact on network performance to analyze linear sensor networks, and most only set a single link model and single optimization target such as the network lifetime. In practice, many algorithms obtain a great delay which isn't applicable. Most papers are only considered in a specific model to get the optimal communication radius by an algorithm without considering the effect of network model, network length, and width on the best network communication radius. In this paper, a mathematical model of the network latency and network lifetime for double-chain linear network is put forward at the first part. The effect process of changes in the communication radius on the network life, network latency is detailed analyzed, and then optimal communication radius algorithm is put forwards based on delay tolerance. At the second part, the paper analyzes the impact of network size, network length, width and other network structure on the optimal communication radius by theoretical and simulation network, optimal configuration 
scheme of communication radius based on network architecture has been obtained.

\section{NETWORK MODEL AND PROBLEM DESCRIPTION}

\section{A. Network model}

For special monitoring environmental such as railways, bridges, roads and other composed of linear topology wireless sensor network, the model is approximately linear and stable. Using precise way to deploy several nodes arranged in a direction along the network on both sides. The actual roads or railways, bridges, etc., are all regional segments, the nodes are generally arranged in the same location of the area, so the paper set up consistent node spacing. Suppose the network length of L, width of $\mathrm{W}$, network size is $\mathrm{N}$, the node spacing of 2D. Assuming the existence of a unique node number $i$, and the communication radius $r$, the network model is shown in Figure 1. BS is the base station:

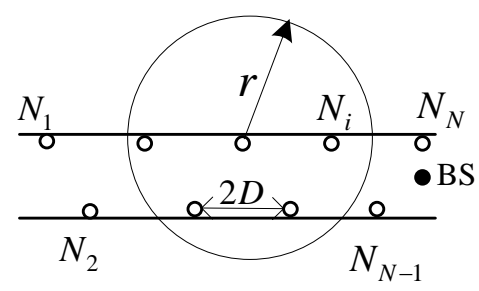

Figure 1. Network topology

Assumptions:

(1) All nodes are distributed in a specific area, namely the node position is determined, each node has a unique identity(ID);

(2) All nodes have the initial energy $E_{0}$. Each node generates $l$ bit data within a single cycle, and eventually sent to base station;

(3) All communication radius is effected by transmit power;

(4) Only consider the energy consumption of data sensing and data transmission, and other energy is too small to calculation;

Based on minimum hops routing protocol, node choose single or multi-hop for transmission.

\section{B. energy consumption model}

Using classic model of energy consumption[9], model of energy consumption between nodes transmit a data as follows,

$$
\left\{\begin{array}{l}
E_{I}=l_{t} E_{\text {elec }}+l_{t} \varepsilon_{f s} d^{2}+l_{r} E_{\text {elec }} i=1,2, \ldots, n \text { if }\left(d<d_{0}\right) \\
E_{I}=l_{t} E_{\text {elec }}+l_{t} \varepsilon_{\text {amp }} d^{4}+l_{r} E_{\text {elec }} i=1,2, \ldots, n \text { if }\left(d>d_{0}\right)
\end{array}\right.
$$

Wherein, $E_{\text {elec }}$ represents the energy loss of transmitting circuit; $l_{t}$ represents data per-node transmitting during unit period; $l_{r}$ is the data per-node receiving; $\varepsilon_{f s}, \varepsilon_{\text {amp }}$ respectively represents the energy required for the two models in the power amplifier.

\section{Problem description}

Few research the specific impact of communication radius on linear wireless sensor networks performance. Under different communication radius, same routing and same network model, the transmission path is different, and then energy consumption and performance are different. This study is the impact of changing the communication radius of double-chain linear topology network model on network performance, to find the optimal communication radius in order to optimize the network lifetime based on delay tolerance. Then the study concerns on the effect of different network widths, length and scale to the optimal communication radius. Configuration scheme of communication radius is proposed under different network structures.

\section{THE ANALYSIS OF DOUBLE-CHAIN MODEL AND CONFIGURATION SCHEME OF COMMUNICATION RADIUS}

\section{The analysis of energy consumption for double-chain model}

As shown in Fig.2, when the communication radius is $r$, using the Minimum Jump routing protocol to transmission $^{[10]}$, the jump distance $d_{i i}$ is the distance from source node $i$ to the nearest node to $\operatorname{sink}$ node $j$ at the coverage of communication range. $d_{i j}=\max \left\{d_{12}\right.$, $d_{13}, \ldots d_{1 i}$, all $\left.d_{i j}<=r\right\}$.

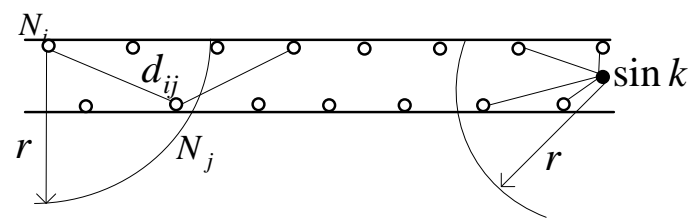

Figure 2. Communication model of double chain type linear topological network

Jump distance is divided into two cases: one is the sink node is covered; one is far from the sink node. In the first case, because of same communication radius, the jump distance is consistent. At final jump, the jump distance is based on the location of the node far from the sink node. So the distance equation can be simplified as follows:

$$
d_{i j}= \begin{cases}\left((0.5 \times W)^{2}+(N-i)^{2} \times d_{N-i}^{2}\right)^{1 / 2} & \text { if } N-i \leq j \\ d_{0} & \text { if } N-i>j\end{cases}
$$

According to Fig. 2, Omitted derived formula, the hop count data from node $\mathrm{i}$ transmitted to sink can be represented as follows:

$$
H_{i j}= \begin{cases}1 & \text { if } N-i \leq j \\ \operatorname{INT}\left(\frac{N-i}{j}+1\right) & \text { if } N-i>j\end{cases}
$$

The amount of data for each node forwards is:

$$
b_{i}=\operatorname{INT}\left(\frac{i-1}{j}\right)+1
$$

Assuming unit amount of data is $l$. Substitute the equation (3) (5) into the formula (1), $E_{i}$ the energy consumption within a unit period can be obtained of each node,

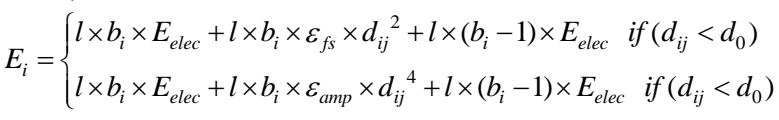




\section{E. The analysis of network latency}

Network latency mainly come from the following three aspects ${ }^{[11]}$, transmission delay model for a single node is as follows:

$$
\text { Del }=D_{\text {proc }}+D_{\text {prop }}+D_{\text {queue }}
$$

Wherein, $D_{\text {proc }}$ represents processing delay, $D_{\text {prop }}$ represents transmission delay, $D_{\text {queue }}$ represents queuing delay. $D_{\text {proc }}$ increases linearly with the number of hops, $D_{\text {proc }}=H_{i} \times \mathrm{C}, \mathrm{C}$ represents units processing delay which is fixed value ${ }^{[12]}$. $D_{\text {prop }}$ depends on the communication distance and network bandwidth. As transmission speed quickly, the delay of communication distance is negligible. $D_{\text {prop }}=l / v_{0} \times H_{i}, \quad v_{0}$ represents the radio channel communication rate. $D_{\text {queue }}$ depends on the access channel rate, the greater the throughput the larger delay node queuing. As shown in Fig.3, there are twice hops, network packets is $b_{1}$ except $b_{2}$. At this competition, for the first time the channel may successful or fail at first time but successful at second time, as a result the max queuing time is $D_{\text {queue }}=0+b_{1} / v_{0}$. In the same way, Fig. (4) shows queuing time of thrice hops, the max queuing time is $D_{\text {queue }}=\sum_{1}^{H_{i}-1} \frac{l}{v_{0}} \times H_{j}$. For the final jump, there are $j$ nodes at the coverage, so $D_{\text {queeu }}=(j+1) \times \frac{l}{v_{0}}$. So the total network latency can be written as:

$$
\operatorname{Del}(i)=H_{i} \times C+l / v_{0} \times H+\sum_{1}^{H_{i}-1} \frac{l}{v_{0}} \times H_{j}+(j+1) \times \frac{l}{v_{0}}
$$

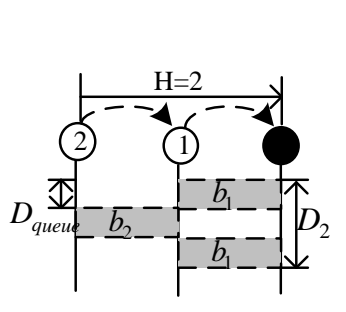

Figure 3. Twice-hop

Network latency model

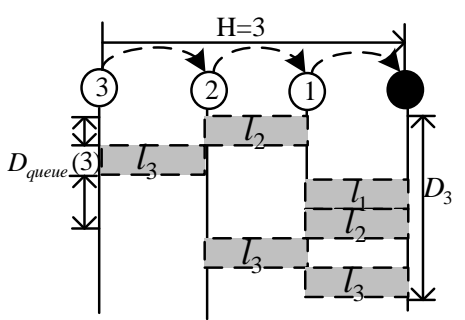

Figure 4. Thrice-hop network latency model
Define $\Gamma$ as tolerance for the network latency, represents the maximum delay permitted by the network expressed as follows:

$$
\Gamma=\min \left\{D e l_{i}\right\}+0.3 \times\left(\max \left\{D e l_{i}\right\}-\min \left\{D e l_{i}\right\}\right)
$$

\section{F. Resolution scheme of optimal communication radius}

Define $E_{\max }$ as the energy consumption of the highest consumption of network node, $E_{\max }=\max \left\{E_{1}, E_{2}, \ldots\right.$, $\left.E_{N}\right\}$. The lifetime of this node effects the overall life of networks, the nodes transmit and receive energy consumption is more than $80 \%$ of overall networks consumption. If researchers only consider this consumption as the energy consumption of network, the network lifetime can be simplified as follows:

$$
T=E_{0} / E_{\max }
$$

Define $D_{\max }$ as the global network latency, $D_{\text {max }}=\max \left\{\operatorname{Del}_{1}, \operatorname{Del}_{2}, \ldots, \operatorname{Del}_{N}\right\}$. Optimization goal is to find the communication radius at the location of maximum network lifetime within tolerance range.

$\max T$

$$
\text { s.t. } \max \left\{\operatorname{Del}_{i}\right\}-\Gamma<0
$$

\section{G. model simulation}

Model parameters are shown in Table 1:

Table 1. Model parameters

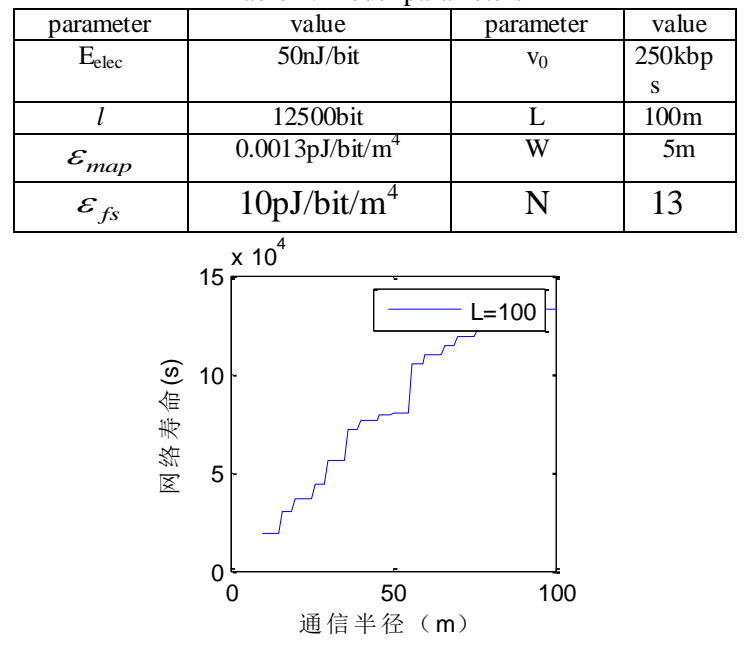

Figure 5. Lifetime of network

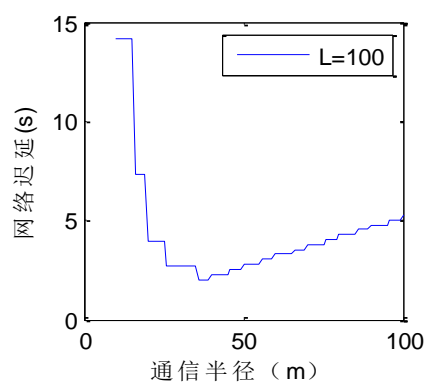

Figure 6. Time delay of network

The above chart shows that, on the basis of meeting the delay tolerance $r$ is $41 \mathrm{~m}$, network lifetime is longer. When selecting communications radius $r=80$ that represents maximum network life, the network latency is high which isn't conducive to enhance network performance. Select combination of factors to be considered, and ultimately choose $r=41 \mathrm{~m}$ as the optimal communication radius.

From the practical consideration, selecting such a program not only can ensure the network lifetime, but also take into account the network latency which has greater impact on network performance for linear topology greater. To some extent, the algorithm enhances the network performance.

\section{THE ANALYSIS OF NETWORK STRUCTURE}

\section{H. The impact of network length}

Node spacing D is constant. Change the distance L and find the optimal communication radius. $(2 \mathrm{D}=10)$ researchers can get: 


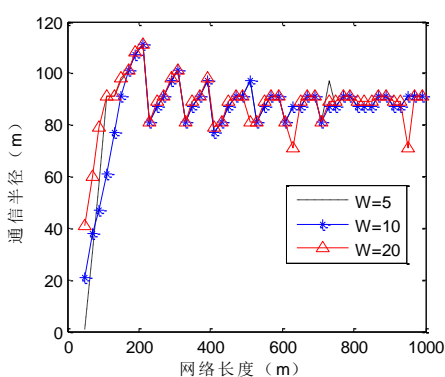

Figure 8. The effect of network length to the optimal communication radius

As shown in Fig. 8, with the increase of L, the optimal communication radius substantially linear increases, and then level off. When $\mathrm{L}$ is relatively small, the three curves do not overlap, indicating that the change of $\mathrm{w}$ has a certain impact on $\mathrm{r}$; When $\mathrm{L}$ is large enough, the three curves are nearly coincident, change of $\mathrm{W}$ hardly influence the selection of r. So in practical project at long distance network, the effect of $\mathrm{W}$ to configure communication radius can be ignored.

\section{The impact of network size}

$\mathrm{W}$ and $\mathrm{L}$ are constant. Change the separation distance D and find the optimal communication radius. ( $L=200$, L=400), represent in Fig. 9 and Fig. 10.

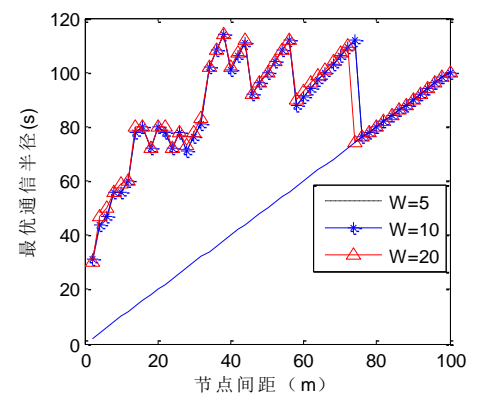

Figure 9. The effect of node space to the optimal communication radius (1)

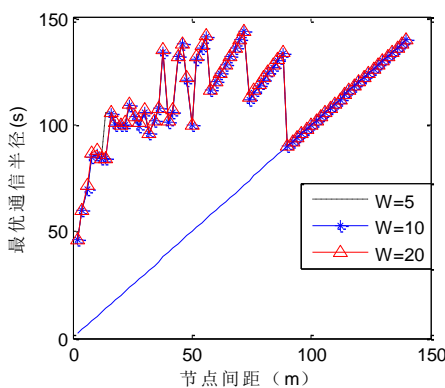

Figure 10. The effect of node space to the optimal communication radius (2)

According to Fig.(9)-(10), Linear networks of different lengths can be obtained the same trend, with the node spacing increases, $r$ increases, and optimal communication radius is greater than the distance of nodes that represents multi-hop transmission. When spaces reach a certain value, the optimal communication radius is close to the node spacing that is to say choosing the single-hop to transmit.

\section{J. The impact of network width}

$\mathrm{L}$ and $\mathrm{D}$ are constant $(\mathrm{D}=1, \mathrm{~L}=20)$. Change $\mathrm{W}$ and seek the optimal communication radius. When the inner width varies at small distance $\mathrm{W}=1 \sim 7 \mathrm{~m}$, analyzing the impact of changing the communication radius on the network performance, researchers can obtain comparative graphs shown as follows:

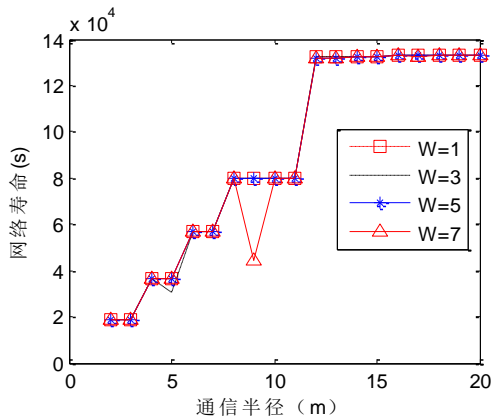

Figure 11. Network lifetime comparison chart

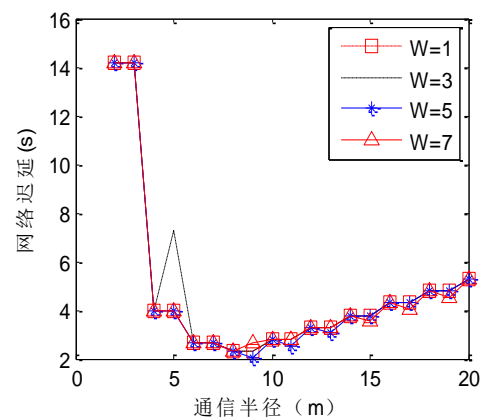

Figure 12. Network time delay comparison chart

As can be seen from the chart, four curves do not overlap and the optimal value isn't the same, indicating that in short-distance transport process, the network width for selecting a communication radius has certain influence. For more intensive spacing, L shorter model, choose a larger radius is beneficial to network performance.

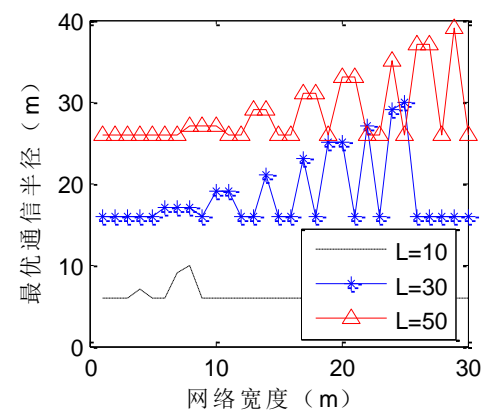

Figure 13. The effect of network width to the optimal communication radius

By analyzing Fig. (11) - (12), researchers can get changing the width $\mathrm{W}$ has an impact on selecting the optimal communication radius at the short-distance transport. When $\mathrm{L}$ is shorter, $\mathrm{W}$ has greater effect on $\mathrm{r}$, and gradually the effect trends to stable. At the small size of the network, $r_{o p}>W$, selecting communication radius that is larger than separation distance is better, which means with two links coordinate to transport can improve the efficiency. When $\mathrm{L}$ is smaller, it tends to choose relatively small communication radius, that is to say, two links without coordination are conducive to enhancing network performance. Conclusion is consistent with the actual project, proving the model correctness. 


\section{K. Configuration scheme of communication radius based on the size of network}

This part analyzes the impact of different parameters on the model. Compared with Literature [5], the proposed model is more applicable to the general situation. The following conclusions can be obtained: When the node spacing is small, multi-hop transmission is beneficial to the network performance. While in large node spacing with large network size, it is conducive to enhance network performance by narrowing the jump distance tending to single-hop transmission. For network length, at the beginning, the optimal communication radius increases with $\mathrm{L}$ almost in linear growth and gradually stabilizes to a certain value. Under the same conditions, when increasing network length, linearly increasing the communication radius could optimize network performance. For network width, when in a relatively small value, width has small impact on network performance, meaning choosing a relatively large communication radius with chains coordinating to transmit is beneficial to enhance network performance. While in a large value, researchers can choose a relatively balanced value to ensure network performance, that is to say, up to a certain extent there are more advantageous to select individual transmission for network performance.

\section{CONCLUSIONS}

For fixed-chain type linear wireless sensor network such as roads, railways, and bridges, many studies have focused on routing protocol research without considering the effect of communication radius. Different communication radius can directly impact on the network transmission and then impact the network performance. From the perspective of practical application, for doublechain model with a minimum jump routing protocol for transmission, the paper crystallizes energy consumption and network latency model to obtain the detailed influence process of change of communication radius for network life, and network latency. The optimal communication radius algorithm is put forwards based on delay tolerance. Then the paper focuses on impact of network length, width, and network size for the optimal communication radius, and summarizes the optimal configuration scheme of communication radius. The simulation results verify the accuracy of the model; the program is consistent with the actual project. In practical applications, the result has certain significance for configuration of communication radius of double-chain linear wireless sensor network such as railways. It is meaning for us to clear understand the transmission of the networks

\section{REFERENCES}

[1] Yick J, Mukherjee B, Ghosal D. Wireless sensor network survey[J]. Computer networks, 2008, 52(12): 2292-2330.

[2] Wang A, Heinzelman W B, Sinha A, et al. Energy-scalable protocols for battery-operated microsensor networks[J]. Journal of VLSI Signal Processing systems for signal, image and video technology, 2001, 29(3): 223-237.

[3] Zhu Y H, Shen D D, Wu W D, et al. Dynamic routing algorithms optimizing lifetime of wireless sensor networks $[\mathrm{J}][\mathrm{J}]$. Acta Electronica Sinica, 2009, 5: 023.

[4] Hossain A, Radhika T, Chakrabarti S, et al. An approach to increase the lifetime of a linear array of wireless sensor nodes[J]. International Journal of Wireless Information Networks, 2008, 15(2): 72-81.

[5] Wang Z, Zhao X, Qian X. An analysis of energy balanced deployment strategy for strip-based wireless sensor networks[C]//Intelligent Control and Automation (WCICA), 2012 10th World Congress on. IEEE, 2012: 4472-4477.

[6] Mao J B, Mao Y M. The analysis of node transmission radius effect of wireless ad hoc network capacity [J].Computer Application, 2008, 25(8): 2491-2494.

[7] Shelby Z, Pomalaza-Raez C, Haapola J. Energy optimization in multihop wireless embedded and sensor networks[C]//Personal, Indoor and Mobile Radio Communications, 2004. PIMRC 2004.15th IEEE International Symposium on. IEEE, 2004, 1: 221-225.

[8] Dong P, Gao S. Adjustment of transmission radius in linear wireless sensor networks[C]//Wireless Communications, Networking and Mobile Computing, 2008. WiCOM'08.4th International Conference on. IEEE, 2008: 1-4.

[9] P Chatterjee. Coverage constrained non-uniform node deployment in Wireless Sensor Networks for load balancing [C]/ /Wireless Communications, Networking and Mobile Computing, 2014 IEEE International Conference on. Kolkata: IEEE, 2014: 213-217.

[10] Han K H, Ko Y B, Kim J H. A novel gradient approach for efficient data dissemination in wireless sensor networks[C]//Vehicular Technology Conference, 2004. VTC2004-Fall. 2004 IEEE 60th. IEEE, 2004, 4: 2979-2983.

[11] Kawadia V, Kumar P R. Principles and protocols for power control in wireless ad hoc networks $[\mathrm{J}]$. Selected Areas in Communications, IEEE Journal on, 2005, 23(1): 76-88.

[12] Wu Y C, Cheung L F, Lui K S, et al. Efficient communication of sensors monitoring overhead transmission lines[J]. Smart Grid, IEEE Transactions on, 2012, 3(3): 1130-1136.

[13] Lindsey S, Raghavendra C S. PEGASIS: Power-efficient gathering in sensor information systems[C]//Aerospace conference proceedings, 2002. IEEE. IEEE, 2002, 3: 3-1125-3-1130 vol. 3.

[14] Heinzelman W B, Chandrakasan A P, Balakrishnan H. An application-specific protocol architecture for wireless microsensor networks[J]. Wireless Communications, IEEE Transactions on, 2002, 1(4): 660-670.

[15] Ali S, Qaisar S B, Felemban E A. A reliable connectivity based node placement strategy in linear and hierarchical wireless sensor networks[C]//Personal, Indoor, and Mobile Radio Communication (PIMRC), 2014 IEEE 25th Annual International Symposium on IEEE, 2014: 763-767. 